\title{
Random lasing from localized modes in strongly scattering systems consisting of macroporous titania monoliths infiltrated with dye solution
}

\section{AUTHOR(S):}

Murai, Shunsuke; Fujita, Koji; Konishi, Junko; Hirao, Kazuyuki; Tanaka, Katsuhisa

\section{CITATION:}

Murai, Shunsuke ... [et al]. Random lasing from localized modes in strongly scattering systems consisting of macroporous titania monoliths infiltrated with dye solution. APPLIED PHYSICS LETTERS 2010, 97(3): 031118.

\section{ISSUE DATE:}

2010-07

URL:

http://hdl.handle.net/2433/147188

\section{RIGHT:}

Copyright 2010 American Institute of Physics. This article may be downloaded for personal use only. Any other use requires prior permission of the author and the American Institute of Physics. The following article appeared in APPLIED PHYSICS LETTERS97, 031118 (2010) and may be found at http://link.aip.org/link/APPLAB/v96/i4/p043305_s1 


\title{
Random lasing from localized modes in strongly scattering systems consisting of macroporous titania monoliths infiltrated with dye solution
}

\author{
Shunsuke Murai, ${ }^{1}$ Koji Fujita, ${ }^{1,2, a)}$ Junko Konishi, ${ }^{1}$ Kazuyuki Hirao, ${ }^{1}$ and Katsuhisa Tanaka \\ ${ }^{1}$ Department of Material Chemistry, Graduate School of Engineering, Kyoto University Katsura, \\ Nishikyo-ku, Kyoto 615-8510, Japan \\ ${ }^{2}$ PRESTO, Japan Science and Technology Agency (JST), 4-1-8 Honcho, Kawaguchi, \\ Saitama 332-0012, Japan
}

(Received 28 May 2010; accepted 23 June 2010; published online 23 July 2010)

\begin{abstract}
We have prepared random lasers that consist of macroporous titania monoliths infiltrated with dye solution and that operate close to light localization regime. When the excitation pulse energy exceeds a threshold, discrete spectral lines ascribed to laser oscillation appear on a featureless emission peak. No pulse-to-pulse variation was observed in the spectral positions of lasing lines, indicating that the lasing modes are localized in the medium. We demonstrated selective excitation of lasing modes by pumping at different positions on the sample. (C) 2010 American Institute of Physics. [doi:10.1063/1.3464962]
\end{abstract}

Random lasing is a phenomenon that occurs in a dielectrically disordered medium with optical gain, wherein light undergoes scattering and amplification simultaneously. ${ }^{1-3}$ The simplest scheme to interpret random lasing is based on diffusive feedback, ${ }^{4}$ which predicts a global narrowing of the emission spectrum above a threshold. Under certain experimental conditions, however, discrete spectral lines (laser spikes) appear on top of the narrowed emission peak, which cannot be explained by a purely diffusive model and indicates the existence of mode structures. The first system showing laser spikes was made of zinc oxide $(\mathrm{ZnO})$ that strongly scatters visible light, ${ }^{5,6}$ and thus a highly localized mode was first proposed as a possible origin of the spikes. However, subsequent experiments clarified that the strong scattering is not necessarily required for the observation of laser spikes, and it is now generally accepted that a diversity of modes can support random lasing. ${ }^{1,7}$ In terms of the spatial extent, the laser spikes can be originated from both the localized modes that are spatially confined in the system, and extended ones that cover a large volume of the system.

Among various systems, the random lasers with strongly localized modes are of particularly interest in a technological sense of fabricating tiny lasers. The straightforward way of obtaining localized modes is to use the highly scattering system, such as gallium arsenide ( $\mathrm{GaAs})$ nanopowders, ${ }^{8}$ compacted nanoparticles of titania $\left(\mathrm{TiO}_{2}\right),{ }^{9}$ and nanostructured gallium phosphide $(\mathrm{GaP}){ }^{10,11}$ Here, it is noted that appearance of laser spikes is not a direct manifestation of the presence of highly localized mode because extended modes can also support laser spikes and yield the similar emission spectra. Measurements of the spatial distribution of laser spikes are thus required for the evaluation of mode extent, but they have been performed only for a limited number of systems having high scattering strengths. van der Molen et al. ${ }^{12}$ fabricated a random laser system of porous $\mathrm{GaP}$ infiltrated with a dye solution, and observed several localized lasing modes with the spatial extent of around $2 \mu \mathrm{m}$. Recently, Fallert et $a l^{7}$ have prepared a $\mathrm{ZnO}$ nanocrystalline-based system, and have found the coexistence of two lasing modes with

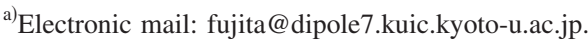

different degree of localization; one is a strongly localized mode with a spatial extent of $5 \mu \mathrm{m}$ and the other a weakly localized mode which spatially spreads out over the system $(\sim 30 \mu \mathrm{m})$.

Although $\mathrm{TiO}_{2}$ is one candidate as a component of a random laser that supports the localized modes because of its high refractive index, the identification of highly localized mode has not been reported for $\mathrm{TiO}_{2}$-based random lasers so far. In this letter, we have fabricated random lasers consisting of macroporous $\mathrm{TiO}_{2}$ monoliths filled with a dye solution and probed the spatial extent of lasing modes, with a particular motivation of making the random laser that operates in the localization regime.

Macroporous $\mathrm{TiO}_{2}$ monoliths were fabricated via sol-gel process accompanied by spinodal-type phase separation. ${ }^{13-16}$ Titanium propoxide, hydrochloric acid, water, N-methyl formamide, and poly(ethylene oxide) with a molecular weight of 10000 were used as starting materials. The as-prepared wet gels were aged and evaporation-dried, and then were heat-treated at $700{ }^{\circ} \mathrm{C}$ to turn the amorphous gel skeleton into a rutile phase. The resultant monoliths possess welldefined throughpores, which have a dual role as a scattering center for visible light and a channel for liquid infiltration. By adjusting the amount of $\mathrm{N}$-methyl formamide in the starting solution, we prepared two samples with central pore sizes of 1.2 and $0.17 \mu \mathrm{m}$, referred to as $\mathrm{TiO}_{2}-1.2$ and $\mathrm{TiO}_{2}-0.17$, respectively. Figure 1(a) shows the differential (indicated by solid curves) and cumulative (dashed curves) pore volumes measured for $\mathrm{TiO}_{2}-1.2$ and $\mathrm{TiO}_{2}-0.17$ using mercury porosimetry. The two samples have the sharp pore size distributions, and the porosity is similar to each other $(\sim 40 \%)$. The inset displays the SEM image of $\mathrm{TiO}_{2}-1.2$, in which well-developed three dimensionally interconnected $\mathrm{TiO}_{2}$ skeletons and pores are obvious in the scale of several micrometers. To evaluate the scattering strength of the sample, coherent backscattering (CBS) was measured by the off-centered rotation technique ${ }^{17}$ using a $488 \mathrm{~nm}$ line from an $\mathrm{Ar}^{+}$laser as a light source.

Our random laser system was prepared by immersing the macroporous $\mathrm{TiO}_{2}$ disk (200 $\mu$ m thick) in a methanol solution of rhodamine $640(\mathrm{R} 640,10 \mathrm{mM})$. The porous $\mathrm{TiO}_{2}$ 

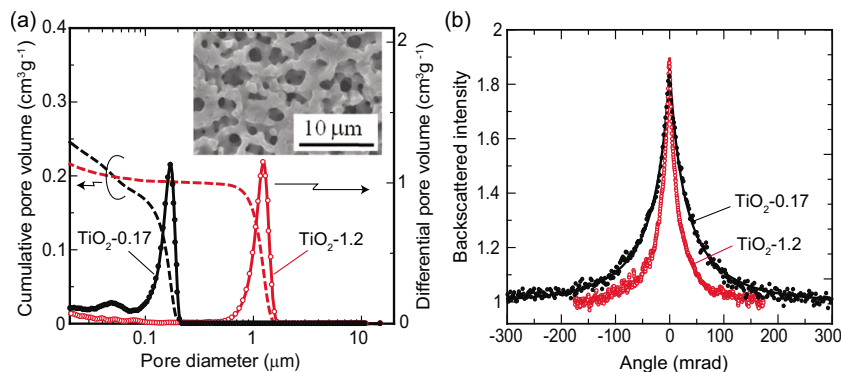

FIG. 1. (Color online) (a) Cumulative (dashed curves) and differential (solid curves) pore size distributions of $\mathrm{TiO}_{2}-0.17$ and $\mathrm{TiO}_{2}-1.2$. Inset shows the SEM image of $\mathrm{TiO}_{2}-1.2$. (b) CBS cones of $\mathrm{TiO}_{2}-1.2$ (open circles) and $\mathrm{TiO}_{2}-0.17$ (closed circles). Solid curves represent the theoretical fits based on the diffusion theory.

sample was attached to the interior wall of a polystyrene cuvette $\left(10 \times 10 \times 40 \mathrm{~mm}^{3}\right)$ using a polystyrene resin to avoid filling of the dye solution between the $\mathrm{TiO}_{2}$ sample and the cuvette, and then was infiltrated with a dye solution. The cuvette was put on the stage equipped with a stepper motor so that the sample could be translated in a direction parallel to the sample surface. The frequency-doubled output $(\lambda=532 \mathrm{~nm})$ of a mode-locked neodymium-doped yttriumaluminum garnet $\left(\mathrm{Nd}^{3+}\right.$ : YAG) laser $(10 \mathrm{~Hz}$ repetition rate and 25 ps pulse width) was used as a pump source. The pump pulse focused by a lens (15 $\mathrm{mm}$ focal length) was incident normal to the sample surface. The diameter of pump spot on the sample surface was estimated by a knife-edge method to be about $30 \mu \mathrm{m}$, which was reasonably larger than the reported typical size of localized mode $(\sim 5 \mu \mathrm{m})$. Photoluminescence (PL) signal was collected at $45^{\circ}$ with respect to the sample surface by a fiber bundle, dispersed by a monochrometer, and detected by a liquid-nitrogen cooled charge coupled device. PL signal was integrated over 50 excitation pulses for each data acquisition.

Figure 1(b) displays the angular resolved CBS cones for porous $\mathrm{TiO}_{2}$ samples. The width of the cone is inversely proportional to the transport mean free path $\ell_{t}$, the length after which the direction of light propagation is randomized. We fit the data with a theoretical curve based on diffusion theory ${ }^{18}$ to estimate $k \ell_{t}$, where $k$ is a wave vector. In the fitting process, the reflectivity of the surface was calculated using the Maxwell-Garnett effective medium theory in order to correct for the overestimation of $k \ell_{\mathrm{t}}$ due to internal reflection. After the correction, the $k \ell_{\mathrm{t}}$ value was estimated to be 15.5 and 5.5 for porous $\mathrm{TiO}_{2}-1.2$ and $\mathrm{TiO}_{2}-0.17$, respectively. We also measured the CBS cone for porous $\mathrm{TiO}_{2}-0.17$ infiltrated with a polymer having the refractive index similar to methanol and obtained $k \ell_{\mathrm{t}} \sim 8.2$.

Figure 2 shows the PL properties for dye-infiltrated porous $\mathrm{TiO}_{2}$ samples. For the neat dye solution, the PL spectrum exhibits a broad spontaneous emission with a maximum at $598 \mathrm{~nm}$ and full width at half maximum (FWHM) of 20 $\mathrm{nm}$, even when excited with a pump power of $220 \mathrm{~nJ} /$ pulse, the highest energy during our experiment [Fig. 2(a), gray curve]. For $\mathrm{TiO}_{2}-1.2$ infiltrated with a dye solution [black curves], a narrowing of PL spectrum occurs when the pump power is increased above $119 \mathrm{~nJ} /$ pulse, and FWHM of the emission becomes about $7.5 \mathrm{~nm}$ [Fig. 2(b)]. The spectral narrowing is accompanied by a rapid increase in emission intensity, showing the presence of threshold for lasing. It should be noted that although the global spectral narrowing
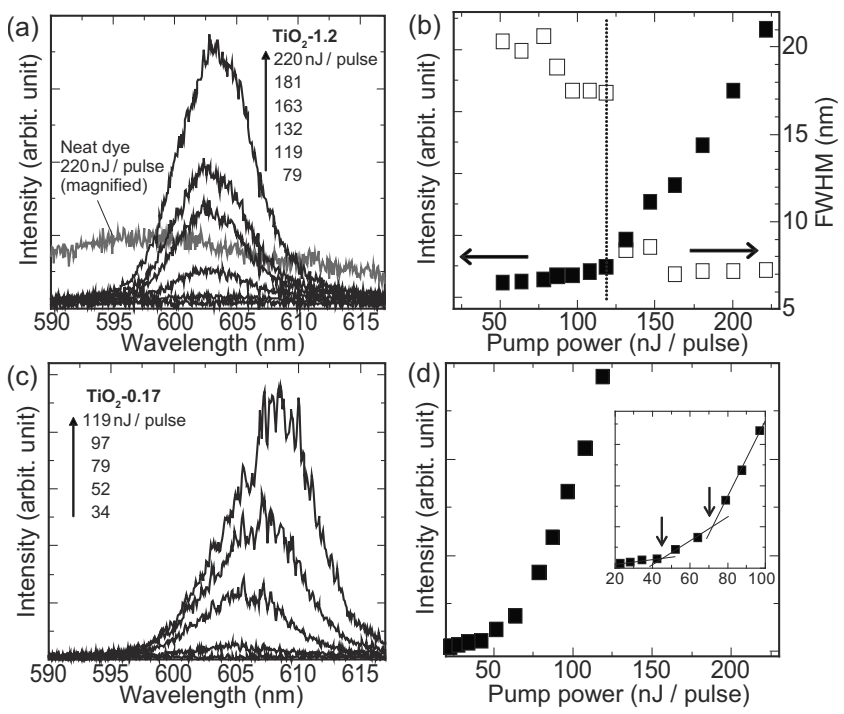

FIG. 2. (a) PL spectra of $\mathrm{TiO}_{2}-1.2$ infiltrated with a R640 solution measured with various pump powers (black solid curves). Emission from a neat R640 solution excited at $220 \mathrm{~nJ}$ is described by a gray solid curve. (b) Pump power dependence of integrated PL intensity (left axis) and FWHM (right axis) for $\mathrm{TiO}_{2}-1.2$ infiltrated with a R640 solution. The vertical dotted line represents a pump power of $119 \mathrm{~nJ} /$ pulse. (c) PL spectra of $\mathrm{TiO}_{2}-0.17$ infiltrated with a R640 solution measured with various pump powers. (d) Pump power dependence of integrated PL intensity for $\mathrm{TiO}_{2}-0.17$ infiltrated with a R640 solution. The inset enlarges the region around the thresholds. Three lines are guides for the eyes, and arrows represent two thresholds at 45 and $75 \mathrm{~nJ} /$ pulse.

occurs, no laser spikes appear in the emission spectrum. In Fig. 2(c), PL spectra under various pump powers are shown for $\mathrm{TiO}_{2}-0.17$ infiltrated with a dye solution. The spectral narrowing of broad emission starts at a much lower pump power compared to $\mathrm{TiO}_{2}-1.2$, and laser spikes emerge on top of the featureless narrowed emission peak when the pump power exceeds $70 \mathrm{~nJ}$. The thresholds for both the global spectral narrowing and the emergence of laser spikes are also recognized in the plot of integrated emission intensity versus pump power [Fig. 2(d)]; one can detect two thresholds at 45 and $75 \mathrm{~nJ}$ as indicated by arrows in the inset. The appearance of laser spikes in the emission spectra is stable for the integration over 50 excitation pulses, indicating that after each excitation pulse, the system lases in the same modes and therefore at the same wavelengths. The observation implies that lasing modes are localized in $\mathrm{TiO}_{2}-0.17$ based system.

To directly investigate the spatial extent of the localized lasing modes, PL spectra for $\mathrm{TiO}_{2}-0.17$ based system were collected while pumping at different positions of the sample. In our experiment, we translated the sample with steps of $1 \mu \mathrm{m}$ in the direction parallel to the sample surface (defined as $x$-axis). The pump power was set to around $97 \mathrm{~nJ}$, which is well above the threshold. In Fig. 3(a), we show the PL spectra collected at different sample positions (from $x=0$ to $8 \mu \mathrm{m})$. Let us discuss the behavior of some representative lasing peaks. The sharp peak at $\lambda=602.05 \mathrm{~nm}$, denoted by (i) in Fig. 3(a), is not observed at $x=0$ and $1 \mu \mathrm{m}$, appears at $x=2,3,4,5$, and $6 \mu \mathrm{m}$, and disappears at $x=7$ and $8 \mu \mathrm{m}$. This means that the mode responsible for the peak (i) can be excited only when $x$ is between 2 and $6 \mu \mathrm{m}$. The similar behavior can be seen for the sharp peak at $\lambda=605.50 \mathrm{~nm}$, denoted by (iii), which appears as a weak emission at $x=0 \mu \mathrm{m}$, grows in intensity at $x=1,2$, and 3 , then decreases at $x=4,5$, and 6, and finally disappears at $x=7$ and $8 \mu \mathrm{m}$. 


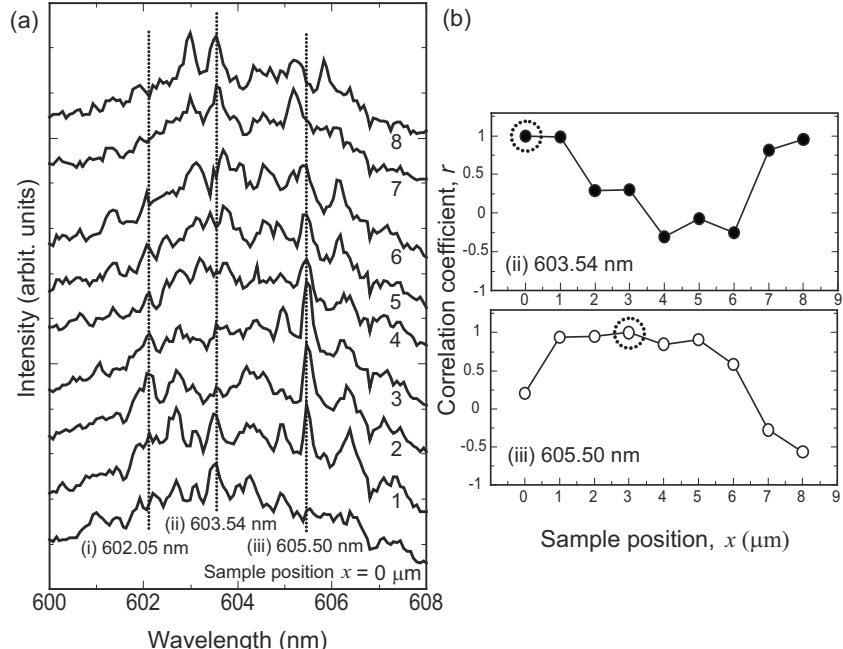

FIG. 3. (a) PL spectra of $\mathrm{TiO}_{2}-0.17$ infiltrated with a R640 solution obtained at various sample positions $x$ while pump power is fixed to $97 \mathrm{~nJ}$. The spectra were translated vertically for clarity. Vertical dotted lines correspond to the wavelengths of the representative lasing peaks: (i) $602.05 \mathrm{~nm}$, (ii) $603.54 \mathrm{~nm}$, and (iii) $605.50 \mathrm{~nm}$. (b) The correlation coefficient $(r)$ between the data taken at a reference position (indicated by dotted circles) and that at various $x$. The $r$ values were calculated from the spectra in Fig. 3(a) after subtracting the broad background emission. Only the data of $\pm 0.35 \mathrm{~nm}$ around the peaks (ii) and (iii) were used for the calculation.

The behavior of these peaks implies that the different modes can be excited by changing the pumping position. Spatial extents of those two modes are on the order of $5 \mu \mathrm{m}$, and are comparable to the values reported for other random lasers that operate in or close to localization regime (e.g., $2 \mu \mathrm{m}$ for a GaP-based system ${ }^{12}$ and $5 \mu \mathrm{m}$ for a $\mathrm{ZnO}$-based system ${ }^{7}$ ).

A close inspection of Fig. 3(a) reveals interesting behavior for the sharp peak at $\lambda=603.54 \mathrm{~nm}$, denoted by (ii). This peak appears at $x=0$ and $1 \mu \mathrm{m}$, becomes less intense or almost disappears at $x=2,3,4,5$, and 6 , and then revivals as intense emission at $x=7$ and $8 \mu \mathrm{m}$. Note that the $x$ positions where this peak becomes weak correspond to the ones where the peak (iii) is intense. To quantify this, we calculated the correlation between the spectral profiles in Fig. 3(a) in the standard way as the Pearson correlation coefficient $(r)$. After subtracting the broadband pedestal, we picked up the data of $\pm 0.35 \mathrm{~nm}$ around the peaks (ii) and (iii) from each spectrum, and calculated $r$ between the data taken at a reference position $[x=0 \mu \mathrm{m}$ for (ii) and $3 \mu \mathrm{m}$ for (iii) $]$ and that at various $x$. The result is shown in Fig. 3(b). One can recognize the mode competition; ${ }^{19,20}$ the positions where $r$ of peak (ii) is high correspond to the positions where $r$ of peak (iii) is low. Most likely, these two modes spatially overlap, and compete for a gain. In the present excitation condition, the total gain available is not large enough for the both modes to lase simultaneously, and thus only one of the modes that win the competition shows a laser peak.

There are several candidates for the origin of localized mode that results in the observed lasing spikes. One plausible candidate is a strong light localization in the sense of Anderson localization ${ }^{21}$ because the present $\mathrm{TiO}_{2}-0.17$ sample infiltrated with dye solution is strongly scattering $\left(k \ell_{\mathrm{t}} \sim 8.2\right)$. Considering the facts that the localization behavior has been observed up to $k \ell_{\mathrm{t}} \sim 4$ for the system without gain $^{9}$ and up to $k \ell_{\mathrm{t}} \sim 6.4 \pm 2.8$ for the system with gain, ${ }^{12}$ strong confinement of lasing modes may be achieved even though $k \ell_{\mathrm{t}}$ is larger than unity, a theoretical criterion for
Anderson localization. Other mechanisms for occurrence of laser spikes are the confinement of lasing modes induced by either optical absorption ${ }^{22}$ or fluctuations in the refractive index. ${ }^{23,24}$ In the former, absorption of emitted photons in the peripheral area of the excitation spot can reduce the effective sample size and thus decrease the spatial extent of lasing modes, while in the latter, the fluctuations in the refractive index create waveguiding structures by chance. All the three mechanisms lead to strongly localized modes that are confined in a volume of the sample.

In summary, we have prepared random lasers consisting of porous $\mathrm{TiO}_{2}$ monoliths and a R640 methanol solution filled in the throughpores. The random laser actions with laser spikes are recognized, which originate from localized modes created due to extremely high scattering strength. By scanning the system with the pump, we have demonstrated selection of the lasing modes to be excited. The result suggests that the porous $\mathrm{TiO}_{2}$-based system is promising for the application to microsized active elements in photonic devices.

The authors thank K. Nakanishi and K. Kanamori at Kyoto University for mercury porosimetry measurements. This study was supported by a Grant-in-Aid for Young Scientist (B, Grant Nos. 22750188 and 22760512) from the Ministry of Education, Culture, Sports, Science, and Technology (MEXT) of Japan.

${ }^{1}$ D. S. Wiersma, Nat. Phys. 4, 359 (2008)

${ }^{2}$ H. Cao, J. Phys. A 38, 10497 (2005).

${ }^{3}$ Solid-State Random Lasers, edited by M. A. Noginov (Springer, New York, 2005).

${ }^{4}$ V. S. Letokhov, Sov. Phys. JETP 26, 835 (1968).

${ }^{5}$ H. Cao, Y. G. Zhao, S. T. Ho, J. Y. Dai, J. Y. Wu, and R. P. H. Chang, Appl. Phys. Lett. 73, 3656 (1998).

${ }^{6}$ H. Cao, Y. G. Zhao, S. T. Ho, E. W. Seelig, Q. H. Wang, and R. P. H. Chang, Phys. Rev. Lett. 82, 2278 (1999).

${ }^{7}$ J. Fallert, R. J. B. Dietz, J. Sartor, D. Schneider, C. Klingshrin, and H. Kalt, Nat. Photonics 3, 279 (2009).

${ }^{8}$ D. S. Wiersma, P. Bartolini, A. Lagendijk, and R. Righini, Nature (London) 390, 671 (1997).

${ }^{9}$ C. M. Aegerter, M. Störzer, S. Fiebig, W. Bührer, and G. Maret, J. Opt. Soc. Am. A 24, A23 (2007).

${ }^{10}$ F. J. P. Schuurmans, D. Vanmaekelbetgh, J. Van de Lagemaat, and A. Lagendijk, Science 284, 141 (1999).

${ }^{11}$ O. L. Muskens, S. L. Diedenhofen, B. C. Kaas, R. E. Algra, E. P. A. M. Bakkers, J. G. Rivas, and A. Lagendijk, Nano Lett. 9, 930 (2009).

${ }^{12}$ K. L. van der Molen, R. W. Tjerkstra, A. P. Mosk, and A. Lagendijk, Phys. Rev. Lett. 98, 143901 (2007).

${ }^{13}$ K. Fujita, J. Konishi, K. Nakanishi, and K. Hirao, Appl. Phys. Lett. 85, 5595 (2004)

${ }^{14}$ J. Konishi, K. Fujita, K. Nakanishi, and K. Hirao, Chem. Mater. 18, 864 (2006).

${ }^{15}$ J. Konishi, K. Fujita, K. Nakanishi, and K. Hirao, Chem. Mater. 18, 6069 (2006).

${ }^{16}$ J. Konishi, K. Fujita, K. Nakanishi, K. Hirao, K. Morisato, S. Miyazaki, and M. Ohira, J. Chromatogr. A 1216, 7375 (2009).

${ }^{17}$ D. S. Wiersma, M. P. Van Albada, and A. Lagendijk, Rev. Sci. Instrum. 66, 5473 (1995).

${ }^{18}$ J. X. Zhu, D. J. Pine, and D. A. Weitz, Phys. Rev. A 44, 3948 (1991).

${ }^{19}$ H. Cao, X. Jiang, Y. Ling, J. Y. Xu, and C. M. Soukoulis, Phys. Rev. B 67, 161101(R) (2003).

${ }^{20}$ H. E. Türeci, L. Ge, S. Rotter, and A. D. Stone, Science 320, 643 (2008)

${ }^{21}$ E. Abrahams, P. W. Anderson, D. C. Licciardello, and T. V. Ramakrishnan, Phys. Rev. Lett. 42, 673 (1979).

${ }^{22}$ A. Yamilov, X. Wu, H. Cao, and A. L. Burin, Opt. Lett. 30, 2430 (2005).

${ }^{23}$ V. M. Apalkov, M. E. Raikh, and B. Shapiro, Phys. Rev. Lett. 89, 016802 (2002).

${ }^{24}$ R. C. Polson and Z. V. Vardeny, Phys. Rev. B 71, 045205 (2005). 